S llia, E Vasilaki, E Blevrakis, AM Spanaki, E Geromarkaki, E Tavladaki, MD Fitrolaki, G Briassoulis. Paediatric Intensive Care Unit, University Hospital of Crete, Heraklion, Greece

Introduction Use of chest ultrasonography as a diagnostic tool and intrapleural fibrinolytic therapy is an alternative to surgical intervention in children with severe pneumonia and parapneumonic loculated effusions.

Objectives To study complicated parapneumonic effusions using bedside chest ultrasonography $(\mathrm{U} / \mathrm{S})$ and intrapleural urokinase in our PICU.

Methods Children with pleural effusion were recruited during 2006-2009(A) and 2010-2012(B). Chest tube was inserted and intrapleural urokinase $(40,000 \mathrm{IU} / \mathrm{kg} /$ dose every 12 hours) was administered. Surgical intervention was reserved for patients with necrotic pneumonia and pulmonary abscess.

Results 16 patients (age 3.8 \pm 0.7 year; males:females 9:7) were diagnosed with significant effusion. Streptococcus pneumoniae and Staphylococcus aureus were more frequently isolated. More patients in period $\mathrm{B}$ experienced severe necrotic pneumonia compared to $\mathrm{A}$ ( 5 vs. 0 ), needed urokinase ( 4 vs. 1 ), or required surgery ( 2 vs. 0 ) $(\mathrm{p}<0.04)$, and had prolonged length of stay $(13 \pm 4$ vs. $5 \pm 1$ days, $p<0.05)$. Five patients received urokinase $(31 \%)$ and $2(12.5 \%)$ required an operative intervention. Patients requiring surgical intervention had longer length of stay ( 21 vs. 5 or $12, p=0.005$ ). There were no significant differences between groups regarding pleural effusion characteristics, amount of drained pleural fluid (130 \pm 1 vs. $406 \pm 76$ vs. $457 \pm 132 \mathrm{ml}$ ), and fever duration after chest tube insertion ( $8 \pm 8$ vs. $2 \pm 1 v s .10 \pm 7$ days, respectively). None of the patients experienced any side effects due to urokinase; all patients discharged in good condition.

Conclusions Aggressive intrapleural fibrinolytic treatment based on bedside chest ultrasonograhy confers significant benefit in effectively treating multi-loculated pleural effusions, reserving surgical intervention for necrotic pneumonia and pulmonary abscesses.

\section{HOSPITAL-ACQUIRED PNEUMONIA IN CRITICALLY-ILL CHILDREN: INCIDENCE, RISK FACTORS, OUTCOME WITH INSIGHT ON EMERGENCY DIAGNOSIS BY MULTIPLEX POLYMERASE CHAIN REACTION}

doi:10.1136/archdischild-2012-302724.0425

\section{MGE Mansour, S Bendary. Ain Shams University, Cairo, Egypt}

Hospital acquired pneumonia (HAP) is the most frequent hospitalacquired infection in critically ill patients. NNIS system reported that HAP accounts for $31 \%$ of all nosocomial infections acquired in medical ICUs. Increasing incidence of infections by antibioticresistant pathogens contributes to higher mortality, longer ICU stay and higher costs. So we aimed to identify the incidence of HAP, its risk factors, and its effects on outcome, we evaluated as well the usefulness of multiplex polymerase chain reaction (m-PCR) as a tool for emergency diagnosis of HAP.

We examined all consecutive admissions to Pediatric ICU from February 2010 to August 2010. Patients were diagnosed to have HAP when their Clinical Pulmonary Infection Score (CPIS) index was more than 6. Blood and endotracheal aspirate (ETA) were tested for bacterial pathogens by microbiological cultures and multiplex PCR simultaneously.

Results Twenty five patients out of 90 admissions (27.7\%) developed HAP during the observation period, with incidence rate of 13 per 1000 patient days and overall mortality of $56 \%$. Gastro-esophageal reflux disease (GERD), mechanical ventilation (MV), endotracheal re-intubation and sedation were the main recorded risk factors for HAP. Multiplex-PCR showed better sensitivity and positive predictive value than bacterial culture for etiological diagnosis of HAP.
Acinetobacter and Klebsiella pneumoniae were the most common identified pathogens.

Conclusion Hospital-acquired pneumonia adversely affects patients outcome in our setting. Moreover, m-PCR permits simultaneous detection of several bacterial pathogens in a single reaction which can optimize the emergency diagnosis of HAP and can improve etiology-directed clinical management of bacterial pneumonia.

\section{VENTILATOR-ASSOCIATED PNEUMONIA IN CHILDREN: A RETROSPECTIVE ANALYSIS}

doi:10.1136/archdischild-2012-302724.0426

S Stabouli, E Volakli, A Violaki, A Tsolaki, M Krommyda, M Sdougka. PICU, Hippokration Hospital, Thessaloniki, Greece

Background and Aims Ventilator-associated pneumonia (VAP) is a common nosocomial infection in PICU patients, defined as nosocomial pneumonia in patients in mechanical ventilation $\geq 48$ hours. We conducted a retrospective study to determine the incidence of VAP and its impact on outcomes in PICU patients.

Methods The medical records of PICU patients admitted to PICU of a tertiary-care hospital from January 2011 to December 2011 were reviewed. Outcomes measures were length of mechanical ventilation and PICU stay, hospital cost and mortality.

Results 127 patients, mean age $4.48 \pm 4.25$ years, $58.3 \%$ boys were enrolled. 27 admissions resulted in development of 31 episodes of VAP, accounting for a VAP rate of 15.33 per 1000 ventilator days. Mean time to diagnosis for the first VAP episode was $14.30 \pm 18.58$ days from initiation of mechanical ventilation. 4 patients developed $2^{\text {nd }}$ VAP episode at $21.40 \pm 17.91$ day of mechanical ventilation. Age, sex, presence of comorbidity and PRISM III score at admission did not differ between patients with VAP and those without VAP. Patients with VAP had significantly longer PICU length of stay (46.04 \pm 43.68 days vs. 9.10 $\pm 9.25, \mathrm{P}<0,001)$, greater needs for mechanical ventilation( $42.26 \pm 43.56$ days vs. $6.90 \pm 7.75, \mathrm{P}<0,001)$, and higher hospital costs for PICU bed excluding treatment cost $(9207.40 \pm 8737.80$ vs. $1820.00 \pm 1850.47, \mathrm{P}<0,001)$, than those without VAP. Patients with VAP presented increased mortality (25.9\% vs. $15 \%)$, but the difference didn't reach statistical significance.

Conclusions VAP in critically ill children is associated with prolonged mechanical ventilation, longer PICU stay and increased hospital cost, emerging the need for effective prevention strategies.

\section{SHORT TERM OUTCOME OF HYALIN MEMBRANE DISEASE IN NEWBORN PERIOD}

doi:10.1136/archdischild-2012-302724.0427

'A Mohammadzadeh, ${ }^{2} \mathrm{~A}$ Farhat, ${ }^{1} \mathrm{~A}$ Heydari, ${ }^{3} \mathrm{H}$ Esmaili, ${ }^{1} \mathrm{M}$ Mahmoodi. ${ }^{1}$ Neonatal Research Center of Mashhad, University of Medical Science; ${ }^{2}$ Neonatology; ${ }^{3}$ Biostatistics, Neonatal Research Center of Mashhad, University of Medical Science, Mashhad, Iran

Background and Aims Hyalin membrane disease (HMD) is one of the most common causes of death and complications in preterm newborn. The incidence is indirectly related to gestational age and birth weight. The aim of this study was to determine short term outcome of the babies' whit HMD.

Methods During one year study, all preterm newborn with diagnosis of HMD admitted to NICU of Emamreza hospital, Mashhad, Iran were elected. Diagnosis was based on clinical symptom, chest $x$ ray, blood gas, and duration of disease. The babies with other cause of respiratory failure, congenital anomaly, surgery problem and transient tacypnea of newborn were excluded. In this NICU nursing to patients' ratio is 1 to 4 and occupied bed ratio is $85 \%$. 